\title{
Competing Memes Propagation on Networks: A Network Science Perspective
}

\author{
Xuetao Wei, Nicholas C. Valler, B. Aditya Prakash, Iulian Neamtiu, Michalis Faloutsos, and Christos Faloutsos
}

\begin{abstract}
In this paper, we study the intertwined propagation of two competing "memes" (or data, rumors, etc.) in a composite network. Within the constraints of this scenario, we ask two key questions: (a) which meme will prevail? and (b) can one influence the outcome of the propagations? Our model is underpinned by two key concepts, a structural graph model (composite network) and a viral propagation model $\left(S I_{1} I_{2} S\right)$. Using this framework, we formulate a non-linear dynamic system and perform an eigenvalue analysis to identify the tipping point of the epidemic behavior. Based on insights gained from this analysis, we demonstrate an effective and accurate prediction method to determine viral dominance, which we call the EigenPredictor. Next, using a combination of synthetic and real composite networks, we evaluate the effectiveness of various viral suppression techniques by either a) concurrently suppressing both memes or b) unilaterally suppressing a single meme while leaving the other relatively unaffected.
\end{abstract}

Index Terms-Epidemics, Competition, Prediction, Suppression

\section{INTRODUCTION}

$\mathbf{I}$ $\mathrm{N}$ THIS PAPER, we examine the competition of two opposed memes across interconnected agents by extend the popular susceptible-infected-susceptible (SIS) compartmental model to construct a novel propagation scheme. We are inspired by the popularity of epidemic models spanning various disciplines. In fact, epidemic models already accurately describe various network spreading phenomena such as the spread of social information, computer viruses, fashion trends, religious beliefs, market penetration and product adoption [1], [2], [3], [4], [5], [6]. Throughout this work, we use the general term "meme" to represent the propagating datum, but, without loss of generality, we may easily substitute the term "computer virus," "flu," or "rumor."

In our scenario, we consider two memes spreading across a population of individuals. A meme's ability to jump from individual to individual is governed by a number of factors unique to the meme itself. Thus, at a network level, the world of each meme is unique. We capture the unique network views of each competing meme through a novel graph structure we

Manuscript received August 16, 2012; revised January 30, 2013.

X. Wei and I. Neamtiu are with UC Riverside (e-mail: \{xwei,neamtiu $@$ cs.ucr.edu)

N. C. Valler is with Crowdcompass, Inc. (e-mail: nvaller@ crowdcompass.com).

B. A. Prakash is with Virginia Tech (e-mail: badityap@cs.vt.edu).

M. Faloutsos is with the Universitiy of New Mexico (e-mail: michalis@ cs.unm.edu).

C. Faloutsos is with Carnegie Mellon University (e-mail: christos@ cs.cmu.edu).

Digital Object Identifier 10.1109/JSAC.2013.130607.

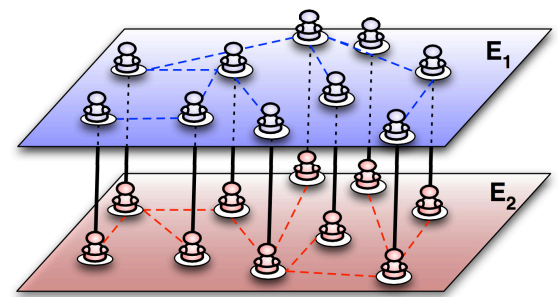

(a) Example Composite Network

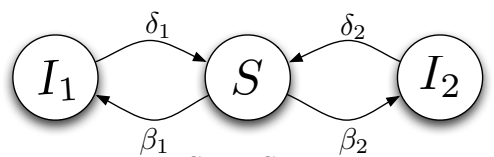

(b) $S I_{1} I_{2} S$ Model

Fig. 1. (a) Example Composite Network topology: a single set of nodes $N$ with two distinct edge sets $E_{1}$ and $E_{2}$. (b) The $S I_{1} I_{2} S$ State Transition Diagram, where $S$ represents the susceptible state and $I_{\{1,2\}}$ indicate the infected state for memes $M_{1}$ and $M_{2}$. The transitions between states are indicated by the directed edges labeled $\beta_{\{1,2\}}$ and $\delta_{\{1,2\}}$.

refer to as a composite network, illustrated in Figure 1(a). Each meme propagates across a unique plane representing a unique connectivity between individuals in our system. A composite network $C$ is a tuple of nodes $N$ and two edge sets $E_{1}, E_{2}$, i.e, $C=\left(N, E_{1}, E_{2}\right)$, where $E_{1} \not \equiv E_{2}$. We further assume that each individual may "possess" a single meme at a time, a constraint we refer to as mutual exclusivity.

The following example can make this problem more concrete. Consider the 2011 Egyptian revolution, which according to reports was partly coordinated via Twitter [7]. To counter such a Twitter campaign, a tech-savvy government could inject confusing and competing information using a malicious Facebook application that spreads to someone's friends. Then, which propagation will win? Hence a focal point of our paper is to predict the winner, by looking at the connectivity and the propagation behavior of its "memes." Naturally, another important question is what means one could use to influence the outcome of these competing information dissemination campaigns.

Interestingly, previous work has not focused on this problem. In fact, most previous efforts either studied a single epidemic on a single topology [8], [9], [10], [11], [12], [13], [3], or studied two pathogens, but on the same topology, and under the assumption that the two viruses appear one after the other [14]. More recent work has studied the quarantining techniques in the case of computer viruses [15], but it has not attempted to study the problem analytically or derive the 
conditions that predict the outcome in the composite networks. We discuss previous work in more detail in $\S \mathrm{VII}$.

In our earlier work [16], we provided a rigorous formulation of competing memes on composite networks using a modified susceptible-infected-susceptible (SIS) propagation mechanism. We also proposed a Non-Linear Dynamic System (NLDS)-based solution for the epidemic threshold, which determines the phase transition of the behavior of the system. This paper subsumes our previous work, and we briefly review the formulation and modeling of the problem for completeness. A key characteristic of our work is that it is applicable for any pair of topologies for the composite network. To validate our work, we use both synthetic composite networks with up to 50,000 of nodes, and a real composite network (specifically, mobile calls and text messages for an enterprise with 235 users for a given mobile carrier). In summary, our main contributions are:

1) We propose EigenPredictor, a method to predict which meme wins. The method relies on the insight from our analytical work mentioned above and uses multinomial logistic regression. A key strength of our method is that it can predict dominance by capturing the complex interplay of the two memes using just the first eigenvalues of appropriately defined system matrices for each meme. In extensive simulations with real and synthetic data, our method can predict the winner (dominating meme) with high accuracy, typically more than $95 \%$.

2) We design and evaluate several suppression strategies based on the insights obtained from our analysis. We focus on two problems: (a) Unilateral Suppression, where we want to quench one meme while allowing the other to progress relatively unimpeded; and (b) Concurrent Suppression, in which we wish to eliminate both memes. Both suppression objectives are constrained by the number of allowed interventions, that is, the maximum number of nodes we can select to suppress the spread of the memes.

3) We evaluate the effect of cross-contamination between the layers of the composite network. In the crosscontamination scenarios, we allow the meme from one network to eventually "transform" into a meme on the other network. For example, think of a rumor propagating in Facebook being transformed into a rumor spread on twitter by an individual user. Cross-contamination adds a new dimension to the dynamic behavior of the competition, and this is arguably the first study that formulates and studies crossing-over.

The rest of the paper is organized as follows. We present the model and problem definitions in Section II and describe the proofs for the threshold in Section III. We present the EigenPredictor in Section IV. We show how to manage propagation and the effect of cross-contamination in section $\mathrm{V}$. We discuss future directions in Section VI. In Section VII we review related work, and Section VIII concludes our paper.

\section{Model And PRoblem Definitions}

\section{A. Our $S I_{1} I_{2} S$ Propagation Model}

We now present our meme propagation model for the competing memes; Table I explains our terminology. The propagation model is based on the popular "flu-like" SIS (Susceptible-Infected-Susceptible) model [8]. We name our model $S I_{1} I_{2} S$ (Susceptible - Infected 1 - Infected I $_{2}$ - Susceptible). Each node in the graph can be in one of three states: Susceptible (healthy), $I_{1}$ (infected by $M_{1}$ ), or $I_{2}$ (infected by $M_{2}$ ). The state transitions are shown in Fig. 1(b).

Meme persistence: $\delta$. If a node is in state $I_{1}$ (or $I_{2}$ ), it recovers on its own with probability $\delta_{1}$ (or $\left.\delta_{2}\right)$. This parameter captures the persistence of the meme in an inverse way: a high $\delta$ means low persistence. Note that we assume that a node can only be infected by one meme, it cannot be infected by the other.

Meme strength: $\beta$. A healthy node gets infected by neighbors that got infected, and the meme strength is captured by $\beta_{1}$ and $\beta_{2}$. This potential infection is passed to a healthy neighbor in the absence of other interactions and we call this potential infection-in-isolation as an attack. In the following, we decide which infection succeeds (infects a susceptible node $i$ ) . Let $C_{1}$ be the number of attacks (each happening with probability $\beta_{1}$ independently) by node $i$ 's neighbors which are in state $I_{1}$ (infected by $M_{1}$ ); similarly, let $C_{2}$ be the number of neighbors infected by $M_{2}$. Then, we have three possible cases for a node in the Susceptible state:

- node $i$ remains in the Susceptible state if $C_{1}=0$ and $C_{2}=0$.

- node $i$ gets infected with $M_{1}$ with probability $\frac{C_{1}}{C_{1}+C_{2}}$.

- node $i$ gets infected with $M_{2}$ with probability $\frac{C_{2}}{C_{1}+C_{2}}$.

\section{B. Problem Definitions}

Given the competing memes model described above, we now define the specific questions and problems that we address in this work. The input for all the problems is as follows:

Basic Input: (1) a composite network's adjacency matrices $\mathbf{A}_{1}$ and $\mathbf{A}_{\mathbf{2}}$; and (2) the competing memes model's parameters: $\beta_{1}, \delta_{1}$ for $M_{1}$ and $\beta_{2}, \delta_{2}$ for $M_{2}$.

Given this basic input, we describe 4 problems, evenly divided into two broad categories: (1) Dominance and Extinction and (2) Meme Control and Suppression.

1) Dominance and Extinction: Our first two problems are motivated by the natural question of predicting the winner of the competition between the two memes.

Problem 1: Epidemic Threshold. Given: The basic input. Find: A condition (threshold values for $\lambda_{1}$ and $\lambda_{2}$ ) under which the memes, either individually or collectively, die-out. This problem deals with the question of the epidemic threshold for our model, since under the threshold, neither meme survives.

Problem 2: Meme Dominance. Given: The basic input.

Find: Under the given set of parameters, which meme will dominate the system? Clearly, this question is more interesting in the case where one or both memes are above the epidemic threshold.

Defining dominance. Intuitively, we say that a meme dominates over the other meme if it manages to capture more 
TABLE I

TERMINOLOGY

\begin{tabular}{|c|l||c|l|}
\hline Symbol & \multicolumn{1}{|c||}{ Definition } & Symbol & \multicolumn{1}{c|}{ Definition } \\
\hline$M_{1}, M_{2}$ & Meme \#1, \#2 & $\mathbf{A}_{\mathbf{1}}, \mathbf{A}_{\mathbf{2}}$ & Adjacency matrices \\
$\delta_{1}, \delta_{2}$ & Meme persistence of $M_{1}, M_{2}$ & $\beta_{1}, \beta_{2}$ & Meme strength of $M_{1}, M_{2}$ \\
$S$ & Susceptible state & $I_{1}, I_{2}$ & Infected state for $M_{1}, M_{2}$ \\
$\mathbf{S}_{\mathbf{1}}, \mathbf{S}_{\mathbf{2}}$ & System matrix for $\mathbf{A}_{\mathbf{1}}, \mathbf{A}_{\mathbf{2}}$, & $\lambda_{1}, \lambda_{2}$ & Largest eigenvalue of $\mathbf{S}_{\mathbf{1}}, \mathbf{S}_{\mathbf{2}}$ \\
& where $\mathbf{S}=(1-\delta) \mathbf{I}+\beta \mathbf{A}$ & & in absolute value. \\
\hline
\end{tabular}

nodes. The definition hides several subtleties, which have to do with the asymptotic behavior of the system, namely what happens as time goes to infinity [12]. However, as we are reliant on simulations, we are forced to adopt a more practical definition. First, we examine the behavior, after a sufficient warm-up period, when the system converges to some relatively stable state (with only small fluctuations of its infected nodes). We repeat our experiments 100 times and observe less than a $2 \%$ variance in the results. Assuming that we are beyond the warm-up period, we say that a meme prevails if it has infected at least $\theta \%$ more network nodes than the other meme. For the results presented here we used $\theta=10$, but we have also experimented with other $\theta$ values and observed similar qualitative results.

2) Meme Control and Suppression: The remaining problems involve our ability to control the outcome of the meme spread and competition.

Problem 3: Unilateral Suppression. Given: The basic input, and a subset of $k$ nodes.

Find: The nodes to suppress, so that we maximize the chances that $M_{2}$ wins. Specifically, we want to find the best nodes to suppress meme $M_{1}$, so that we favor $M_{2}$. This problem is relevant in a market penetration of competing products, creating buzz in blogs, or a virus-versus-antivirus propagation problem in a computer or epidemiological setting.

Problem 4: Concurrent Suppression. Given: The basic input, and subset of $k$ nodes.

Find: The best nodes to suppress that cause both memes to die-out. That is, we want to find the best set of nodes that reduce the spreading effectiveness of both memes. As an example of concurrent suppression, imagine the spread of two distinct, yet equally false rumors propagated through two distinct fringe pseudo-political groups that share memberships. Due to the potential damage such rumors may cause, both will be suppressed by preventing critical members from spreading the falsehoods.

\section{THE EPIDEMIC THRESHOLD}

In this section, We want to analytically determine the epidemic threshold (Problem 1). First, a discrete-time NonLinear Dynamical System (NLDS), whose general form is $\mathbf{p}_{t+1}=g\left(\mathbf{p}_{t}\right)$, is used to approximate the infection process. The NLDS gives the evolution of the system with time, as we explain below. First, the probability that node $i$ is infected by neighbor node $j$ with meme $M_{1}$ at time $t$ is $\beta_{1} P_{j}^{1}(t-1)$. Then, the probability $\zeta_{i}^{1}(t)$ that node $i$ does not receive the infection of $M_{1}$ from its neighbors (here, we assume the neighbors are independent) as:

$$
\zeta_{i}^{1}(t)=\Pi_{j \in i^{\prime} \text { sneighbors }}\left(1-\beta_{1} P_{j}^{1}(t-1)\right)
$$

Thus, we have the probability that node $i$ receives the infection of $M_{1}$ at time $t$ from its neighbors is:

$$
1-\zeta_{i}^{1}(t)=1-\Pi_{j \in i^{\prime} \text { s neighbors }}\left(1-\beta_{1} P_{j}^{1}(t-1)\right)
$$

With the same reasoning, we can derive the probability of that node $i$ receives the infection of $M_{2}$ from its neighbors at time $t$ is:

$$
1-\zeta_{i}^{2}(t)=1-\Pi_{j \in i^{\prime} \text { s neighbors }}\left(1-\beta_{2} P_{j}^{2}(t-1)\right)
$$

Now, we have the probability that node $i$ is infected by $M_{1}$ from its neighbors at time $t$ is the probability that node $i$ receives the infection of $M_{1}$ and does not receive infection of $M_{2}$ from its neighbors at time $t$ (assuming that the $\beta$ and $\delta$ values are all extremely small, or, equivalently, the time between state transitions is extremely small.) Thus, we get:

$$
T_{i}^{1}(t)=\left(1-\zeta_{i}^{1}(t)\right) \cdot \zeta_{i}^{2}(t)
$$

Using the same reasoning, we have the probability that the node is infected by $M_{2}$ at time $t$ is:

$$
T_{i}^{2}(t)=\left(1-\zeta_{i}^{2}(t)\right) \cdot \zeta_{i}^{1}(t)
$$

Hence the probability that node $i$ is in state $I_{1}$ is:

$$
P_{i}^{1}(t)=\left(1-\delta_{1}\right) \cdot P_{i}^{1}(t-1)+T_{i}^{1}(t) \cdot S_{i}(t-1)
$$

and the probability that it is in state $I_{2}$ is:

$$
P_{i}^{2}(t)=\left(1-\delta_{2}\right) \cdot P_{i}^{2}(t-1)+T_{i}^{2}(t) \cdot S_{i}(t-1)
$$

and the probability that it is in state $S$ (Susceptible) is:

$$
S_{i}(t)=\left(1-T_{i}^{1}(t)-T_{i}^{2}(t)\right) S_{i}(t-1)+\delta_{1} P_{i}^{1}(t-1)+\delta_{2} P_{i}^{2}(t-1)
$$

As mentioned before, for $M_{1}$ we define the vector $\vec{P}^{1}(t)=$ $\left(P_{1}^{1}(t), P_{2}^{1}(t), \ldots, P_{N}^{1}(t)\right)^{\prime}$ where $P_{i}^{1}(t)$ is the probability that node $i$ is infected by meme $M_{1}$ at time $t$. Similarly, for $M_{2}$, we have $\vec{P}^{2}(t)=\left(P_{1}^{2}(t), P_{2}^{2}(t), \ldots, P_{N}^{2}(t)\right)^{\prime}$. Let $\vec{V}(t)=$ $\left(\vec{P}^{1}(t), \vec{P}^{2}(t)\right)$ be the concatenation of two vectors. We use the NLDS formulation to describe the whole infection process evolution as $\vec{V}(t)=f(\vec{V}(t-1))$, with: 


$$
f_{i}(\vec{V}(t-1))=\left\{\begin{array}{cc}
\left(1-\delta_{1}\right) P_{i}^{1}(t-1)+ & \\
T_{i}^{1}(t) S_{i}(t-1) & \text { if } i \leq N \\
\left(1-\delta_{2}\right) P_{i}^{2}(t-1)+ & \\
T_{i}^{2}(t) S_{i}(t-1) & \text { if } i>N
\end{array}\right.
$$

Substituting $T_{i}^{1}(t), T_{i}^{1}(t)$ and $S_{i}(t-1)$ into equation 8 , we find that $f_{i}(\vec{V}(t-1))$ is equal to the following:

$$
=\left\{\begin{array}{cc}
\left(1-\delta_{1}\right) P_{i}^{1}(t-1)+\left(1-\zeta_{i}^{1}(t)\right) \zeta_{i}^{2}(t) & \\
\left(1-P_{i}^{1}(t-1)-P_{i}^{2}(t-1)\right) & \text { if } i \leq N \\
\left(1-\delta_{2}\right) P_{i}^{2}(t-1)+\left(1-\zeta_{i}^{2}(t)\right) \zeta_{i}^{1}(t) & \\
\left(1-P_{i}^{1}(t-1)-P_{i}^{2}(t-1)\right) & \text { if } i>N
\end{array}\right.
$$

We use the following theorem about the asymptotic stability of an NLDS at a fixed point:

Theorem 1 (Hirsch and Smale, 1974 [17]): The system given by $\mathbf{p}_{t+1}=g\left(\mathbf{p}_{t}\right)$ is asymptotically stable at an equilibrium point $\mathbf{p}^{*}$, if the eigenvalues of the Jacobian $J=\nabla g\left(\mathbf{p}^{*}\right)$ are less than 1 in absolute value, where

$$
J_{k, l}=\left[\nabla g\left(\mathbf{p}^{*}\right)\right]_{k, l}=\left.\frac{\partial p_{k, t+1}}{\partial p_{l, t}}\right|_{\mathbf{p}_{t}=\mathbf{p}^{*}}
$$

The fixed point we are interested in for analyzing the threshold is the point where no node is infected (all nodes are healthy), i.e., $\vec{V}^{*}=\overrightarrow{0}$. By using this, we have the following theorem:

Theorem 2: The system is asymptotically stable at $\vec{V}^{*}=\overrightarrow{0}$ if the first eigenvalue of the system matrices for both memes as defined in Table I, are less than 1, i.e., $\lambda_{1}<1$ and $\lambda_{2}<1$, where $\lambda_{1}$ is the largest eigenvalue of matrix $S_{1}=\left(1-\delta_{1}\right) I+$ $\beta_{1} A_{1}$ (and similarly for $\lambda_{2}$ ).

Proof: We are interested in the stability of the fixed point $\vec{V}^{*}=\overrightarrow{0}$. Let the Jacobian at this point be $\nabla(f)($ a $2 N \times 2 N$ matrix). Then

$$
[\nabla(f)]_{i j}=\frac{\partial f_{i}(\vec{V}(t-1))}{\partial \vec{V}_{j}(t-1)}
$$

Next, we can write it into a block matrix composed of the system matrices:

$$
\nabla(f)=\left[\begin{array}{l|l}
S_{1} & S_{3} \\
\hline S_{4} & S_{2}
\end{array}\right]
$$

In order to find the first eigenvalue of $\left.\nabla(f)\right|_{\vec{V}_{f}}$, we define $\vec{X}$ as 2 N elements vector:

$$
\vec{X}=\left[\frac{\vec{X}_{1}}{\vec{X}_{2}}\right]
$$

where $\vec{X}_{1}$ and $\vec{X}_{2}$ have $\mathrm{N}$ elements respectively. We then have:

$$
\left.\nabla(f)\right|_{\overrightarrow{v_{f}}} \vec{X}=\left[\begin{array}{c|c}
S_{1} & S_{3} \\
\hline S_{4} & S_{2}
\end{array}\right] \cdot\left[\begin{array}{c}
\overrightarrow{X_{1}} \\
\overrightarrow{X_{2}}
\end{array}\right]=\left.\lambda_{\nabla(f)}\right|_{\overrightarrow{v_{f}}}\left[\begin{array}{c}
\vec{X}_{1} \\
\vec{X}_{2}
\end{array}\right]
$$

Doing the matrix multiplications, we get:

$$
S_{1} \vec{X}_{1}+S_{3} \overrightarrow{X_{2}}=\left.\lambda_{\nabla(f)}\right|_{\overrightarrow{v_{f}}} \overrightarrow{X_{1}}
$$

and

$$
S_{4} \vec{X}_{1}+S_{2} \vec{X}_{2}=\left.\lambda_{\nabla(f)}\right|_{v_{f}} \vec{X}_{2}
$$

with $S_{1}=\left(1-\delta_{1}\right) I+\beta_{1} A_{1}, S_{2}=\left(1-\delta_{2}\right) I+\beta_{2} A_{2}$ and $S_{3}=S_{4}=0$ (where $I$ is the $N \times N$ identity matrix), as we show in Table I and as discussed below. Hence, the Jacobian $\nabla(f)$ is a block diagonal matrix and its eigenvalues are the same as the eigenvalues of $S_{1}$ and $S_{2}$. So the largest eigenvalue of $\nabla(f)$ can be either $\lambda_{1}$ or $\lambda_{2}$.

\section{EIGENPREDiCTOR: WhO Wins?}

In this section, we determine which meme will prevail in the composite network, which we described as Problem 2. We showed in the previous section that when the system is below the threshold, both memes die-out. Hence, the use of the predictor is meaningful when the parameters are such that at least one of the memes is above threshold and Theorem 2 does not apply.

Due to the complexity of the problem, instead of an analytical solution, we present a predictive model, which we call EigenPredictor, which allows us to predict which meme will eventually prevail in the composite network. We also use simulations with synthetic and real data, which we describe below.

\section{A. Simulation Set-up and Datasets}

A discrete-time simulation of our system is used to simulate the stochastic behavior on different synthetic and real composite networks.

1) Small-scale Data sets ( $N<1,000)$ : Real-world enterprise composite network (ENT). This dataset, which was caputured over the course of six months, represents the phone call and SMS text message communications in one enterprise. Each node is an employee $(|N|=235)$, the edges in $E_{1}$ correspond to SMS messages exchanged between employees, and edges in $E_{2}$ correspond to phone calls made between employees. In detail, of all users, $31 \%$ communicate via calls alone, $28 \%$ via SMS alone, and $41 \%$ via both calls and SMS. In addition to the SMS and phone communication data, ENT also provides a basic social structure among the company's employees. This social information forms the basis of our Social Hierarchy method, which we will describe in Section $\S \mathrm{V}$. Each identified employee is grouped into one of 5 anonymous job roles. Job roles are ordered by their importance, thus we can determine who are the "bosses" and who are the regular employees [16].

Synthetic composite networks. Two synthetic graphs with 1,000 nodes are created: an Erdős-Rényi graph and a scalefree graph; we use the Barabási-Albert model [18]. Several different combinations of topologies have been experimented. Here, the reasons that we focus on these two are : (a)In order to show that our methods are not tailored to a particular family of graphs and (b) scale-free graphs are known to emerge in complex human and communication networks [18].

2) Large-scale Data Sets $(1,000<N<50,000)$ : In order to further stress-test the accuracy of our framework, we also did experiments on synthetic social networks with $1,000<$ $N<50,000$ nodes that are generated by the forestFire, 
randomWalk, and nearestNeighbor graph generation models [19], which are informed by real world measurements of social networks and provide graph structures that resemble such networks.

3) Simulation runs: We use a combination of Matlab and Python to conduct the experiments on real and synthetic composite networks. In each experiment, each meme infects a unique set of nodes $I n i_{1}$ and $I n i_{2}$. Each set of nodes has the same size, and is selected uniformly at random from $N$. The set of nodes is subject to the constraint $I n i_{1} \cap I n i_{2}=\emptyset$ (i.e., mutually exclusive). We run each simulation until it reaches a relatively stable state as we discussed in Section II. At which point, we determine the average number of nodes infected by $M_{1}$ and $M_{2}$ and report the outcome, which then gets averaged across 100 runs.

Accuracy. To measure the accuracy of our model, we compute, for each simulation, the percentage of runs where the outcome (as predicted by EigenPredictor) and the actual result (from the simulation) coincide.

\section{B. The EigenPredictor}

We now describe our EigenPredictor method for predicting which meme prevails. At stable state, we have three possible outcomes, which we represent as follows. If the outcome is ' 1 ', it means that $M_{1}$ will eventually prevail in the composite networks; if the outcome is ' 2 ', it means that $M_{2}$ will prevail; for the case where the difference is less than $\theta$, which here is $10 \%$, the outcome is ' 3 '. Note that we experimented with other $\theta$ values $(5 \%, 10 \%$, and $15 \%)$ and the results were qualitatively similar. To summarize, given the parameters of our system, i.e., $A_{1}, \beta_{1}, \delta_{1}, A_{2}, \beta_{2}, \delta_{2}$, EigenPredictor produces a numeric value $(1,2$, or 3$)$, with the following semantics:

$$
\begin{aligned}
& \text { EigenPredictor }\left(\left(A_{1}, \beta_{1}, \delta_{1}\right),\left(A_{2}, \beta_{2}, \delta_{2}\right)\right) \\
& =\left\{\begin{array}{cl}
1, & \text { if } M_{1} \text { prevails } \\
2, & \text { if } M_{2} \text { prevails } \\
3, & \text { if no clear winner }
\end{array}\right.
\end{aligned}
$$

Since $\lambda_{1}$ is the first eigenvalue (in absolute value) of system matrix $S_{1}$ for $M_{1}$, where $S_{1}=\left(1-\delta_{1}\right) I+\beta_{1} A_{1}$ (and similarly for $\lambda_{2}$ ), then $\lambda_{1}$ is a function of parameters $A_{1}, \beta_{1}, \delta_{1}$ (and the same for $\lambda_{2}$ ). Therefore, equation 9 could be simplified and written as:

$$
\text { EigenPredictor }\left(\lambda_{1}, \lambda_{2}\right)= \begin{cases}1, & \text { if } M_{1} \text { prevails } \\ 2, & \text { if } M_{2} \text { prevails } \\ 3, & \text { if no clear winner }\end{cases}
$$

We use our EigenPredictor in two scenarios: first, when $\lambda_{1}$ and/or $\lambda_{2}$ are below the threshold (Case 1); second, when both $\lambda_{1}$ and $\lambda_{2}$ are above the threshold (Case 2).

Case 1: At Least One Eigenvalue Below Threshold. From Section III, we know that if the system matrix's first eigenvalue of one meme is less than 1, the corresponding meme will die-out eventually. Therefore, in this scenario, we can predict which meme prevails eventually using the following three rules:

(i) if $\lambda_{1}<1$ and $\lambda_{2}>1$, then $M_{2}$ tends to prevail eventually in the composite networks; (ii) if $\lambda_{1}>1$ and $\lambda_{2}<1$, then $M_{1}$ tends to prevail eventually in the composite networks;

(iii) if $\lambda_{1}<1$ and $\lambda_{2}<1$, then both memes will die out and none of them can be said to prevail.

Figures 2(a)-(e) demonstrate the proposed rules on both synthetic and real composite networks. The infection starts by infecting 30 nodes for each meme in Figure 2(a), Figure 2(b) and Figure 2(c), and 10 nodes for each meme in both Figure 2(d) and Figure 2(e). The outcomes of belowand above-threshold from these rules can be distinctly seen in these figures. These results show that, though simple, our proposed rules are very effective for predicting which meme tends to prevail eventually in the composite networks.

Case 2: Both Eigenvalues Above Threshold. This is the more interesting case in terms of competition: each meme in isolation would not die-out, so it is a "fight for dominance." We find again that the system eigenvalues play a critical role: the meme whose first eigenvalue is larger tends to prevail eventually in the composite networks.

Intuitively, the first system eigenvalues capture the likelihood of success for each meme. Recall that the system eigenvalue considers both the topology and the meme strength. Extensive experimental results, shown in Figure 2(f), argue in favor of this observation: we plot the outcome of the competition for different pairs of the eigenvalues $\left(\lambda_{2}\right.$ vs. $\left.\lambda_{1}\right)$; we see that above the diagonal $M_{2}$ prevails, and below the diagonal $M_{1}$ prevails, in other words, the meme with the largest system eigenvalue wins.

Our regression model. We verify the significance of $\lambda_{1}$ and $\lambda_{2}$ as determining factors using a rigorous regression model. In particular, we use a multinomial logistic regression [20] to predict the outcome in the case where both eigenvalues are above the threshold:

$$
\log \frac{\operatorname{Pr}\left(Y=i \mid X=\left(\lambda_{1}, \lambda_{2}\right)\right)}{\operatorname{Pr}\left(Y=K \mid X=\left(\lambda_{1}, \lambda_{2}\right)\right)}=\alpha_{i 0}+\alpha_{i 1} \cdot \lambda_{1}+\alpha_{i 2} \cdot \lambda_{2}
$$

Here $Y$ represents the outcome of this model (dependent variable), and $X$ represents the input, i.e., the independent variables $\lambda_{1}$ and $\lambda_{2} ; \vec{\alpha}_{i}=\left(\alpha_{i 0}, \alpha_{i 1}, \alpha_{i 2}\right)$ is the coefficient vector of independent variables, where $i=1,2$. Therefore, we have:

$$
\begin{aligned}
& \operatorname{Pr}\left(Y=i \mid X=\left(\lambda_{1}, \lambda_{2}\right)\right)= \\
& \frac{\exp \left(\alpha_{i 0}+\alpha_{i 1} \cdot \lambda_{1}+\alpha_{i 2} \cdot \lambda_{2}\right)}{1+\sum_{i=1}^{K-1} \exp \left(\alpha_{i 0}+\alpha_{i 1} \cdot \lambda_{1}+\alpha_{i 2} \cdot \lambda_{2}\right)} \\
& \operatorname{Pr}\left(Y=K \mid X=\left(\lambda_{1}, \lambda_{2}\right)\right)= \\
& \frac{1}{1+\sum_{i=1}^{K-1} \exp \left(\alpha_{i 0}+\alpha_{i 1} \cdot \lambda_{1}+\alpha_{i 2} \cdot \lambda_{2}\right)}
\end{aligned}
$$

where $\mathrm{i}=1,2$ and $\mathrm{K}=3$. The coefficients vectors $\alpha_{i}$ computed by our regression model are presented in Table III; note that corresponding coefficients' values (e.g., $\alpha_{10}$ and $\alpha_{20}$ ) are not, as one might expect at first, equal in magnitude and of opposite signs, since the output range is $1-3$.

To construct the ground truth, we ran system simulations (input: $\lambda_{1}$ and $\lambda_{2}$; output: which meme prevails) on both synthetic and real composite networks. The number of data points was 5,339 for synthetic composite networks and 6,844 data points for real composite networks (see Section IV-A for 


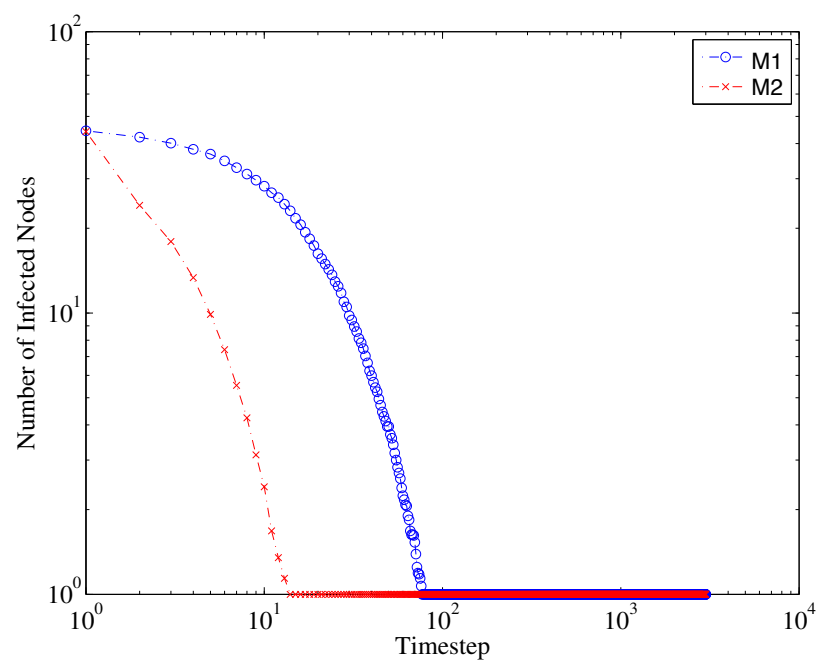

(a)

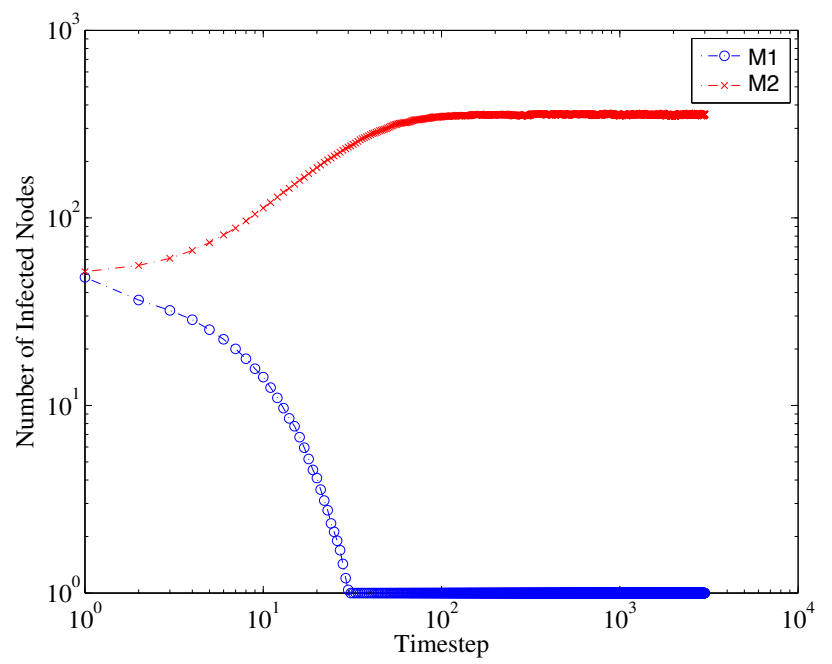

(c)

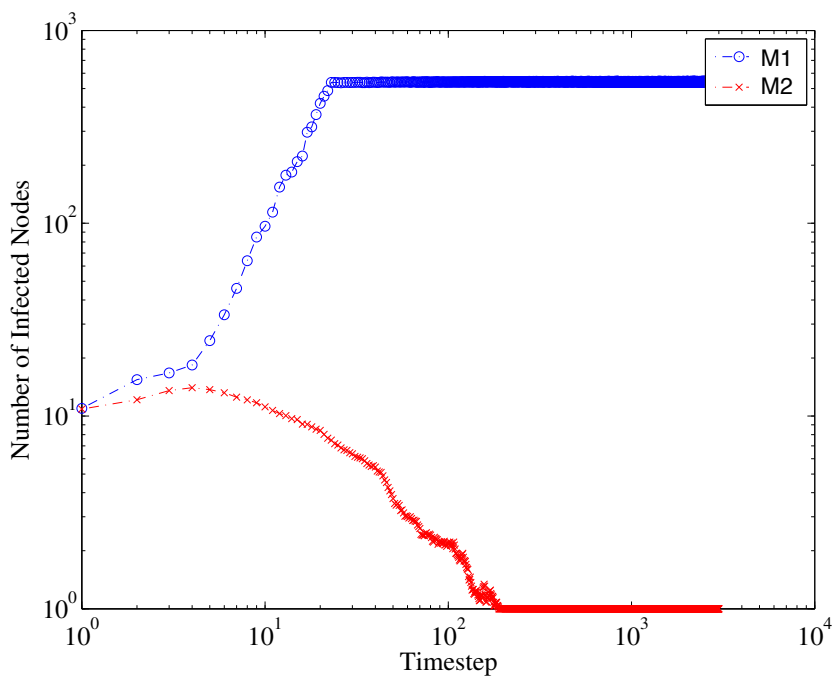

(e)

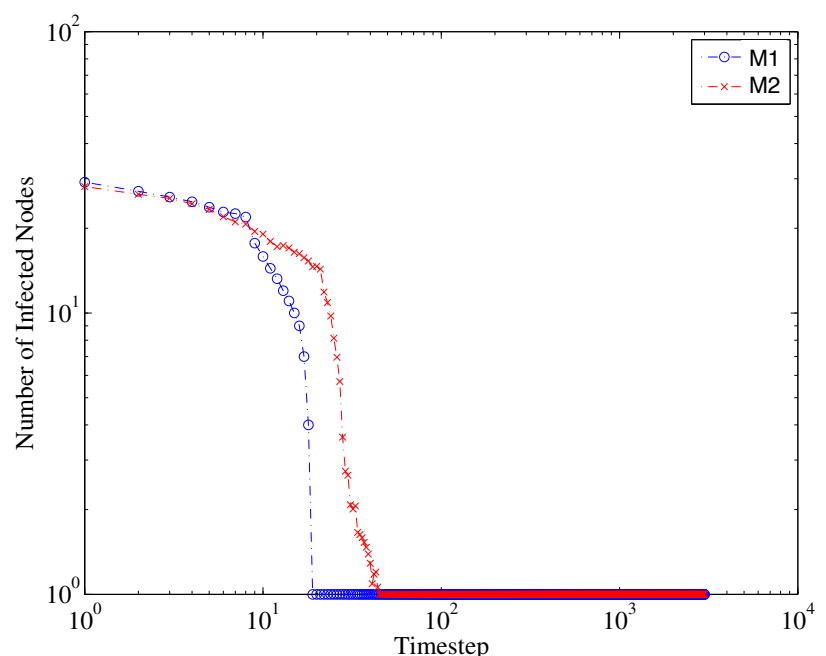

(b)

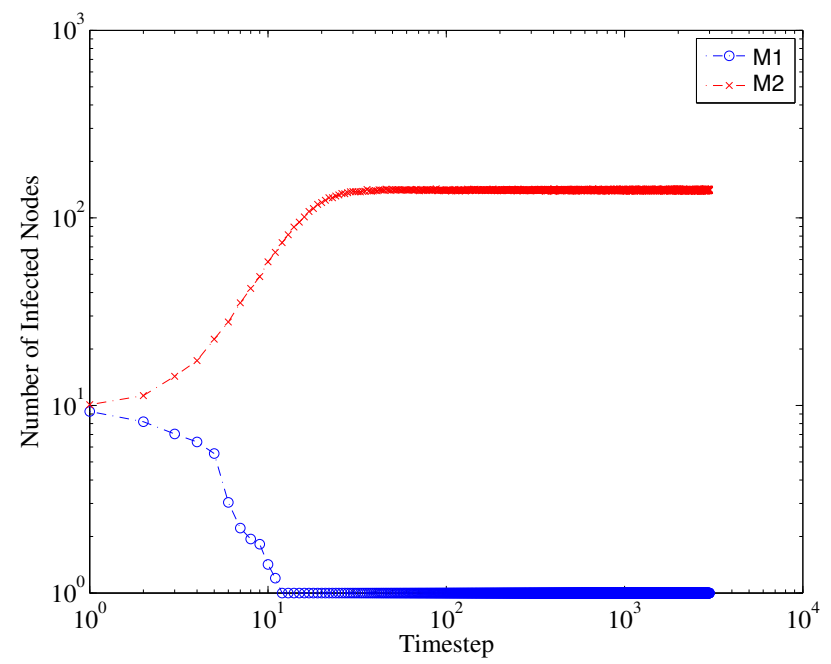

(d)

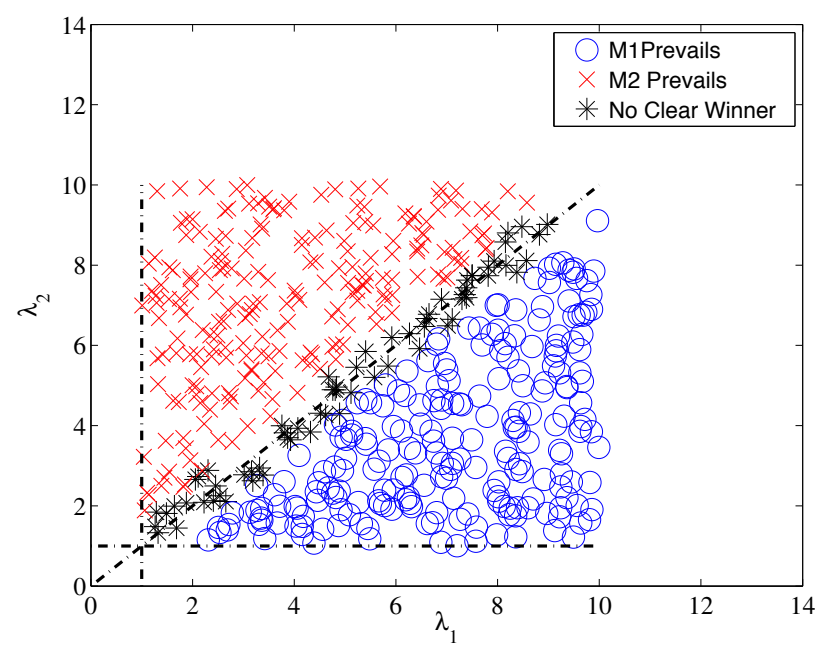

(f)

Fig. 2. Simulation Results: Infection plot over time (log-log) in Figure(a)-(e). 2(a): Synthetic Composite Networks: $\lambda_{1}=0.97, \lambda_{2}=0.96 ; 2$ (b): Real Composite Networks: $\lambda_{1}=0.9, \lambda_{2}=0.94 ; 2$ (c): Synthetic Composite Networks: $\lambda_{1}=0.91, \lambda_{2}=1.63 ; 2(d)$ : Real Composite Networks: $\lambda_{1}=0.99, \lambda_{2}=$ 1.4; 2(e): $\lambda_{1}=4.5, \lambda_{2}=1.7 ; 2$ (f): The outcomes for different combinations of system eigenvalues: $1<\lambda_{1}<10$ and $1<\lambda_{2}<10$; black dotted lines represent three lines $\lambda_{1}=1, \lambda_{2}=1$, and $\lambda_{1}=\lambda_{2}$. When the eigenvalues are roughly equal there is no clear winner. 
TABLE II

PREDICTION ACCURACY

\begin{tabular}{|c||c||c|}
\hline \multirow{2}{*}{$\begin{array}{c}\text { Training data } \\
\text { set size }\end{array}$} & \multicolumn{2}{c|}{ Accuracy } \\
\cline { 2 - 3 } & $\begin{array}{c}\text { Synthetic } \\
\text { networks }\end{array}$ & $\begin{array}{c}\text { Real } \\
\text { networks }\end{array}$ \\
\hline $5 \%$ & $96.65 \%$ & $95.05 \%$ \\
\hline $10 \%$ & $98.42 \%$ & $98.28 \%$ \\
\hline
\end{tabular}

a description of the datasets). Next, for each data point, we compare the outcome from the simulation with the outcome from the EigenPredictor (regression), and compute the accuracy of the predictor.

As shown in Table III, using only 5\% of the dataset as training data (and $95 \%$ of the dataset for validation), our model achieves a prediction accuracy of $96.65 \%$; when using $10 \%$ for training, the accuracy is $98.42 \%$, with $95 \%$ confidence interval.

Stress-testing EigenPredictor: topology independence. In order to test the robustness of our model, we use a different training topology from the testing topology. Specifically, we use $5 \%$ and $10 \%$ of the data set from synthetic composite networks as training data to predict the data set from real composite networks. We achieve an accuracy of $95.05 \%$ and $98.28 \%$, respectively, with a 95\% confidence interval, as shown in Table II. These results indicate that EigenPredictor is very effective in practice.

We now present details on the statistical results and significance of our model: Table III shows the results of the logistic regression $\left(\vec{\alpha}_{1}, \vec{\alpha}_{2}\right.$, and $|t|$-values). To understand how well the independent variables $\lambda_{1}$ and $\lambda_{2}$ contribute to the model (i.e., explain the dependent variable $Y$ ), note the $|t|$-values; since the $|t|$-values are much larger than 2.08 (which translates to $p$-values being much lower than $<0.01$ ), this indicates that our model is statistically significant at the $1 \%$ level. These results indicate that all features contribute to the prediction model, and the model has high predictive power.

Discussion. The significance of the multinomial logistic regression is two-fold: (a) we verify quantitatively that the $\lambda_{1}$ and $\lambda_{2}$ are statistically sufficient to determine the winning meme and (b) we develop a practical method to predict meme dominance under complex dynamics.

\section{Meme Suppression, Control And CROSS-CONTAMINATION}

In this section, we first design and evaluate suppression methods based on two distinct strategies (described as problems 3 and 4 in $\S I I-B 2)$ :

1) Unilateral Suppression. The goal of this strategy is to suppress one meme, while leaving the other unscathed, thus free to spread unimpeded. Using the five techniques described below, we intend to suppress $M_{1}$ by removing ability to spread the memes from a subset of carefully selected nodes. The intention of unilateral suppression is to reduce $\lambda_{1}$ to below $\lambda_{2}$ (i.e., $\lambda_{1}<\lambda_{2}$ ), thus affecting the outcome.

2) Concurrent Suppression. The goal of this strategy is to suppress the spread of both memes by removing a set

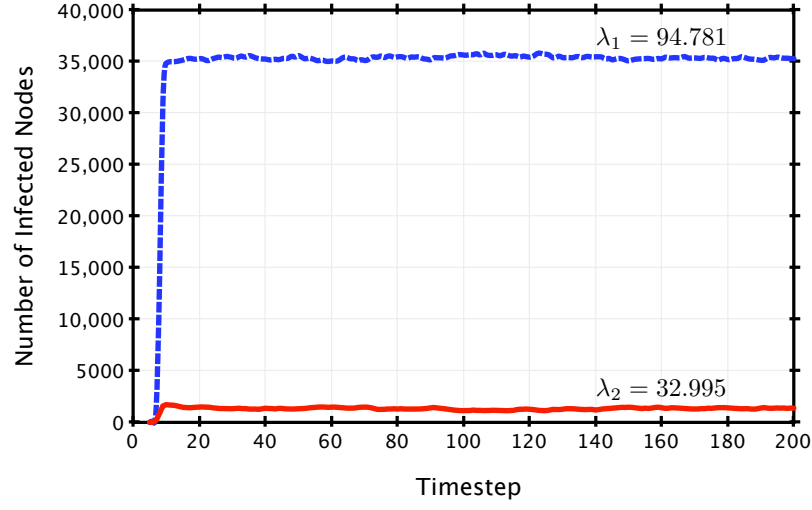

(a) $\lambda_{1}>\lambda_{2}$

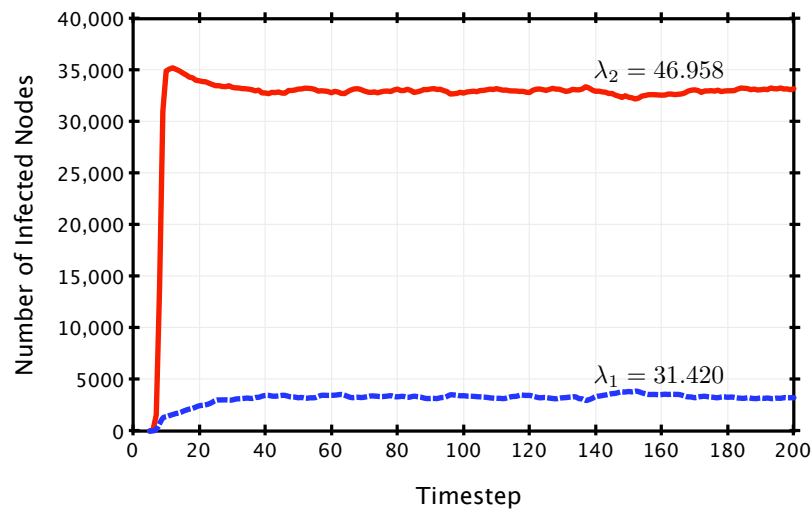

(b) $\lambda_{1}<\lambda_{2}$

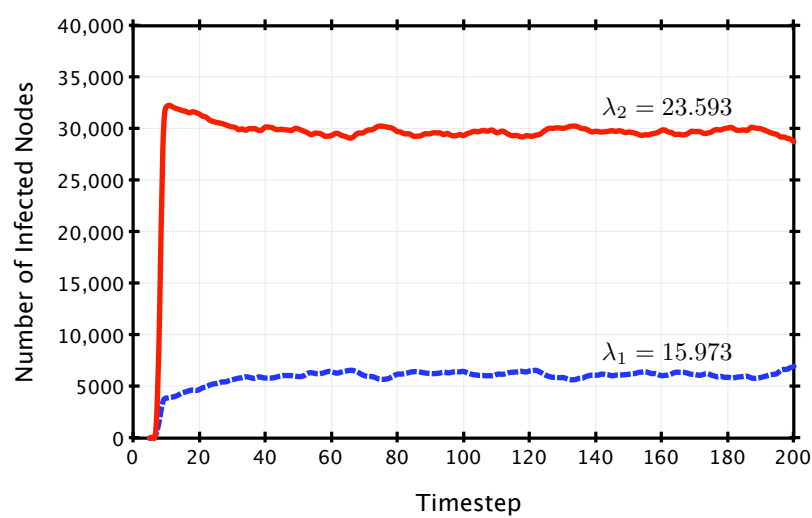

(c) $\lambda_{1}<\lambda_{2}$

Fig. 3. Large Scale Experimental Results. This figure shows experimental results of large scale epidemic simulations using a ForestFire and Nearest Neighbor synthetic graph models. Results above are for $N=40,000$ nodes, but are consistent for results of 10,000 to 50,000 node experiments. Unlike smaller-scale experiments, these results show that the weaker meme may retain some endemic population, yet the meme with the larger eigenvalue clearly dominates the simulation.

of nodes from both graphs in the composite network. Ultimately, we want to reduce $\lambda_{1}$ and $\lambda_{2}$ to below 1 (i.e., $\lambda_{1}, \lambda_{2}<1$ ), thus stopping the spread of both memes.

Given these two main strategies, we propose 5 methods, described in Table IV, partially motivated by the methods used in single virus/disease propagation on a single network [21], [22]. We evaluate each method's effect on 
TABLE III

REGRESSION RESULTS: COEFFICIENT VECTORS AND THEIR CORRESPONDING $|t|$-VALUES.

\begin{tabular}{||l|l|l|l|}
\hline & $\alpha_{i 0}$ & $\alpha_{i 1}$ & $\alpha_{i 2}$ \\
\hline$\vec{\alpha}_{1}$ & -3.43 & 1.44 & -1.32 \\
\hline$|t|$-values for $\vec{\alpha}_{1}$ & 9.27 & 12.18 & 8.66 \\
\hline$\vec{\alpha}_{2}$ & 7.05 & 1.37 & 2.39 \\
\hline$|t|$-values for $\vec{\alpha}_{2}$ & 6.64 & 9.64 & 12.48 \\
\hline
\end{tabular}

the system matrix eigenvalues for each subgraph in the composite network $\left(\lambda_{1}, \lambda_{2}\right)$. The proposed methods are: (a) Random, (b) Acquaintance, (c) Max Degree, (d) Social Hierarchy and (e) Greedy.

\section{A. Unilateral Suppression}

As mentioned above, the objective of unilateral suppression is to reduce $\lambda_{1}$ to less than $\lambda_{2}$, thus reversing the prediction of our EigenPredictor. That is, we seek to answer: What set of nodes should we suppress in order to reduce the spread of one meme, ultimately resulting in the dominance of the other, unsuppressed, meme? We present the results of using Unilateral Suppression on the enterprise data set in Figure 4. Note that $M_{1}$ will eventually prevail in the composite network prior to applying any unilateral suppression strategies. Then, observe that the value of $\lambda_{1}$ decreases as nodes are removed from the system. At $k=10, \lambda_{1}$ is reduced to below $\lambda_{2}$ (thus reversing the prediction of the EigenPredictor).

As expected, the two methods that rely on randomness (i.e., Random and Acquaintance) have the worst performance compared to the other methods. In contrast, Greedy performs better than the others, yet is the most expensive computationally. Max Degree performs surprisingly well, within $1 \%$ of Greedy at much lower computational cost.

Interestingly, when we remove nodes based on their social status (e.g., remove "bosses" before "managers," and so on), the method Social Hierarchy performs better than the random methods, yet not as well as the topologicallyinformed models, and eventually (at $k=20$ ) crosses the value of $\lambda_{2}$. Though not as effective, in situations where we lack topological information, we could potentially rely on easily observable social hierarchy information to inform our suppression process.

\section{B. Concurrent Suppression}

Under the concurrent suppression scheme, the goal is to reduce the effective spreading power of two memes spreading through different modes of communication (i.e., edge sets $E_{1}$, $E_{2}$ in a composite network). Simply put, we ask: What set of shared nodes should we inoculate in order to reduce the spread of both memes the most?

We present the results of our suppression methods in Figure 5. As before, we observe that Max Degree and Greedy reduce both $\lambda_{1}$ and $\lambda_{2}$ to below the epidemic threshold (indicate by the horizontal line at 1 ) at approximately $k=19$.

Social Hierarchy provides mixed results. As indicated by the lower plotted line (representing the SMS portion of the enterprise network), Social Hierarchy is nearly

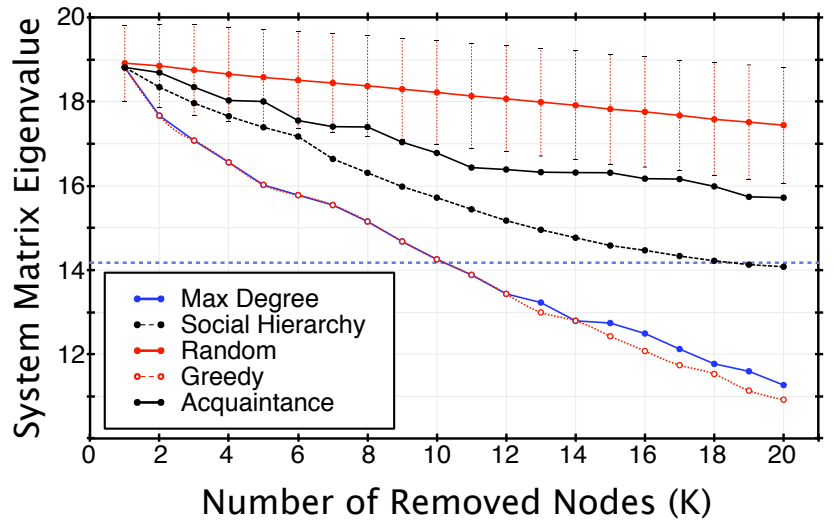

(a)

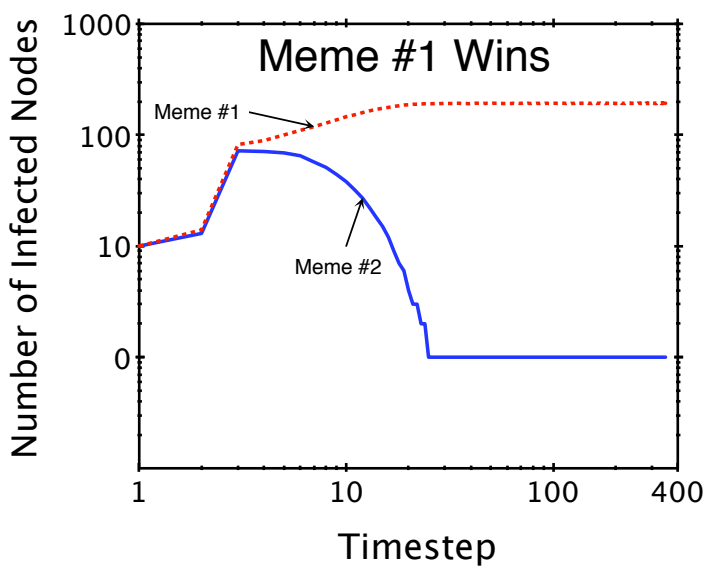

(b) Before Suppression

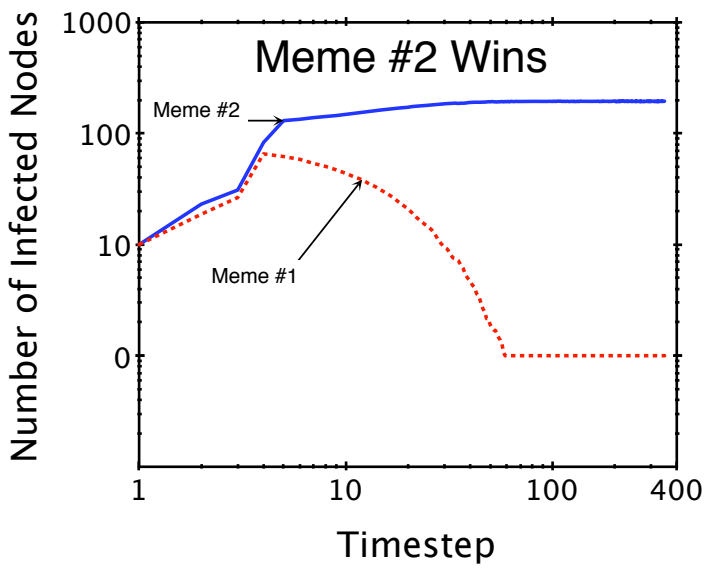

(c) After Suppression

Fig. 4. Example of Unilateral Suppression on the enterprise data set. The methods Greedy, Max Degree and Social Hierarchy drop the system matrix eigenvalue $\lambda_{1}$ below $\lambda_{2}$ (thus reversing the prediction of the EigenPredictor); (b) shows the original competition results without removing nodes; note that $M_{1}$ wins, while $M_{2}$ dies out; (c) shows the competition results after removing $k=20$ nodes using the Max Degree method; the result is reversed, with $M_{2}$ winning and $M_{1}$ dying out.

as effective as Max Degree and Greedy. Yet, on the upper line (call graph of the enterprise network), the Social Hierarchy method is not as effective and does not reduce the spreading power to below the epidemic threshold. 
TABLE IV

SUPPRESSION METHODS

\begin{tabular}{|c|c|c|c|}
\hline Method Name & Unilateral & Concurrent & Intuition \\
\hline Random & $\operatorname{rand}\left(\operatorname{node}_{G_{1}}\right)$ & $\operatorname{rand}\left(\right.$ node $\left._{G_{1} \mid G_{2}}\right)$ & Randomly select a node and remove it from $G_{1}\left(G_{1} \mid G_{2}\right)$ \\
\hline Acquaintance & $\begin{array}{l}\operatorname{rand}(\text { neighbor }) \quad \text { of } \\
\operatorname{rand}(\text { node })\end{array}$ & $\begin{array}{l}\operatorname{rand}(\text { neighbor }) \\
\text { of } \quad \operatorname{rand}(\text { node }) \text { of } \\
\operatorname{rand}\left(G_{1} \mid G_{2}\right)\end{array}$ & $\begin{array}{l}\text { Acquaintance immunization, remove a random neighbor of } \\
\text { a randomly selected node in } G_{1}\left(G_{1} \mid G_{2}\right) \text {. }\end{array}$ \\
\hline Max Degree & $\max \left(\operatorname{deg}\left(G_{1}\right)\right)$ & $\max \left(\operatorname{deg}\left(G_{1} \mid G_{2}\right)\right)$ & Remove node with the maximum degree in $G_{1}\left(G_{1} \mid G_{2}\right)$. \\
\hline $\begin{array}{l}\text { Social } \\
\text { Hierarchy }\end{array}$ & $\max (\operatorname{rank}($ node $))$ & $\max (\operatorname{rank}($ node $))$ & Remove node with the highest rank. \\
\hline Greedy & $\max \left(\lambda_{1}\right)$ & $\overline{\max \left(\lambda_{1} \mid \lambda_{2}\right)}$ & $\begin{array}{l}\text { Remove the node that causes the largest eigenvalue drop in } \\
\text { either } \lambda_{1} \text { or } \lambda_{2} \text {. }\end{array}$ \\
\hline
\end{tabular}

\section{Summary}

In summary, we have designed and evaluated several techniques for unilateral and concurrent suppressions, which are based on randomness, topological information and social hierarchy. The results from both suppressions show that the topological properties-based method (i.e., Max Degree) is very effective in controlling meme propagation compared to other methods. Put another way, removing the highestconnected node is a very effective suppression strategy. In situations where we lack topological information, we could potentially rely on the explicit information of social hierarchy to design our suppression scheme (e.g., Social Hierarchy), though not as effective as the topological properties-based method.

\section{Cross-Contamination Experiments}

Until this point, we have considered memes spreading on a composite network to be mutually exclusive, i.e., a meme will spread using only the edges associated with their own network. In this section, we evaluate the effect of cross-contamination across various synthetic graphs. We change the model slightly: we allow the meme from one network to eventually "transform" into a meme that can propagate on the edges of the other network. For example, consider a rumor propagating in Facebook being transformed into a rumor spread on twitter by an individual user. The user creates a new meme and releases it on twitter, which spreads the same information, but now this meme is spreading across twitter edges. Our simulation model emulates this exact scenario, by carefully following the propagation of these "cross-over" meme. To account for the new ability of a meme to jump composite network layers, each meme is assigned a cross-contamination parameter, denoted $0<X_{A \rightarrow B}, X_{B \rightarrow A} \leq 1.0$. Specifically, $X_{A \rightarrow B}$ describes the ability of a meme propagating on composite network layer $A$ to cross to layer $B . X_{A \rightarrow B}$ is similarly defined.

In the shown scenarios, if simulated in isolation, each of the memes would propagate and capture the graph. While in competition, in each of these simulations, ultimately one meme dominates. We have established that the meme propagating with the largest eigenvalue will eventually dominate the graph in the absence of cross-over. However, the crossing over allows the meme with higher such likelihood to spill over and propagate on the other topology, thus giving an advantage to the meme with higher such likelihood.
In Figure 6(a), we demonstrate the results of cross contamination on a composite network of 500 nodes. In this example, $\lambda_{S, 1}=26.43$, and $\lambda_{S, 2}=13.24$ with cross-contamination probabilities of $X_{A \rightarrow B}=.15$ and $X_{B \rightarrow A}=0.05$. Observing that $\lambda_{S, 1}>\lambda_{S_{2}}$, we see that meme \#1 ultimately captures the greatest number of nodes. Interestingly, meme \#2 does not completely die out, possible due to the ability of meme \#2 to cross over to both layers of the composite network.

In Figure 6(b), we show the average steady-state percentage of infected nodes versus the propagations strength of meme $\# 2\left(\beta_{2}\right)$. All other parameters are constant, in particular $\beta_{1}=0.25, X_{A \rightarrow B}=0.10$, and $X_{B \rightarrow A}=0.10$. We observe that meme \#2 will eventually dominate the simulation as we increase $\beta_{2}$. Due to space limitations, we cannot present a more extensive study of this case, despite having more results, while it would be interesting to study this problem analytically.

\section{DISCUSSION AND FUtURE WORK}

In this section, we discuss the limitations of our work and possible future directions.

Choice of epidemic model. The flu-like SIS (SusceptibleInfected-Susceptible) epidemiological model is simple, yet illustrative, and has been extensively studied in past literature in a single-virus setting (cf. [9], [1], [23]). Therefore, we chose to extend SIS in order to gain fundamental insights into the dynamics of competing memes. We leave the investigation of other epidemic models as future work.

Using real composite networks. Finding real data for any networked system or communication is non-trivial due to privacy concerns, infrastructure limitations, and measurement biases. Finding real data sets of composite networks is even more challenging. Obtaining the enterprise dataset used in this paper was instrumental in modeling and understanding how real composite network operate, but the dataset comes with dissemination restrictions. We believe that the research community could greatly benefit from the creation of an open repository of real composite networks.

Deeper exploration. Our paper is the first attempt to study, predict and manage competing epidemic propagations on composite networks. We leave further exploration, like finding the extent of foot-prints, proving performance bounds for inoculation policies and incorporating more elaborate interactions between the two networks as future work. 


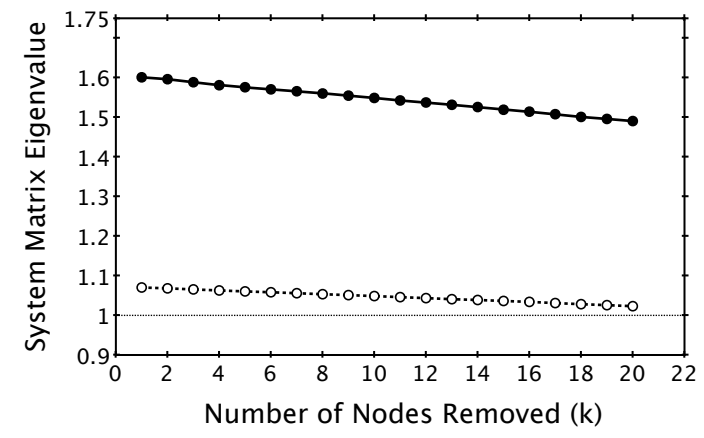

(a) Random

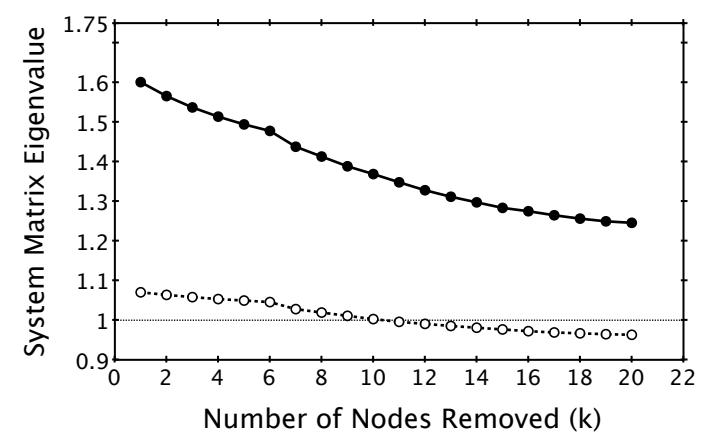

(b) Social Hierarchy

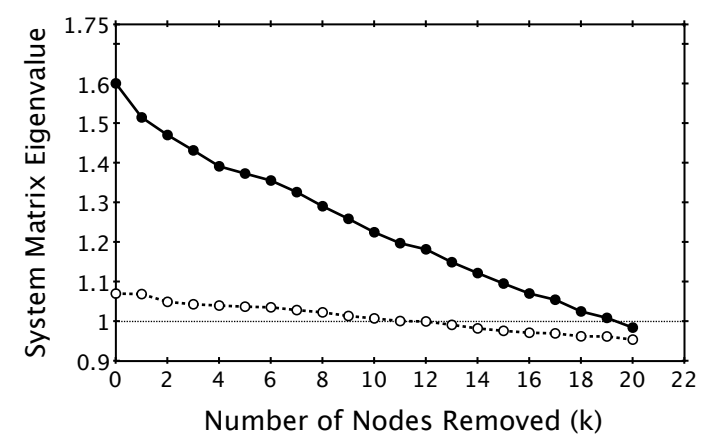

(c) Max Degree

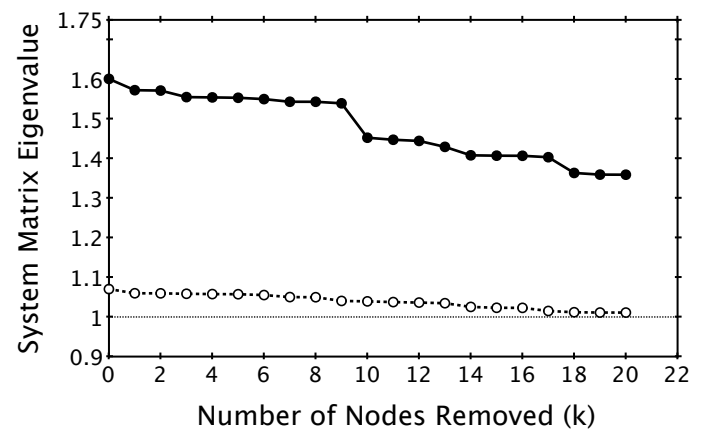

(d) Acquaintance

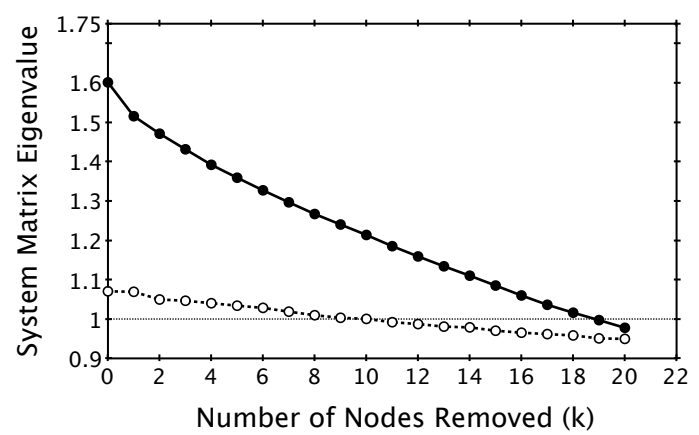

(e) Greedy

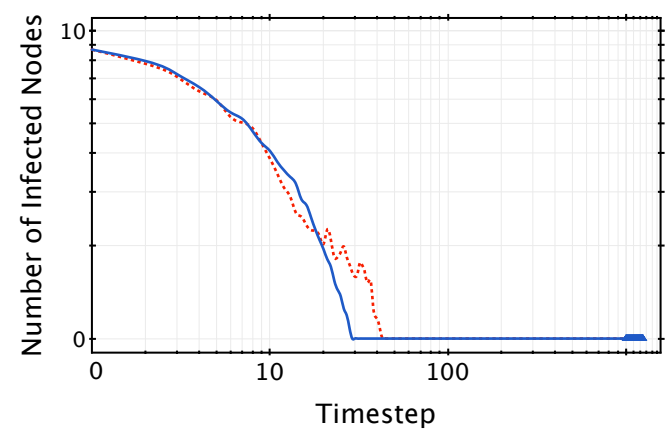

(f) Outcome of Concurrent Suppression

Fig. 5. Example of Concurrent Suppression on the enterprise data set, using each method. The epidemic threshold is marked in each plot at 1 . Again, both the Greedy and Max Degree methods drop $\lambda_{1}$ and $\lambda_{2}$ below the epidemic threshold. Subplot 5(f) shows suppression results after removing $k=20$ nodes selected using the Max Degree method-both memes die out.

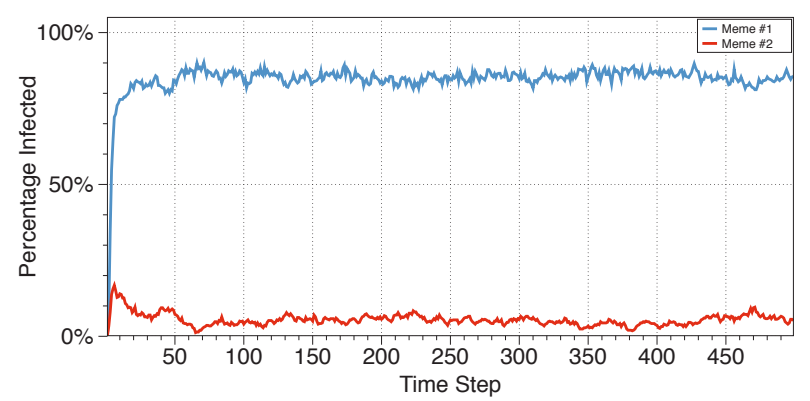

(a) Example of Cross-Contamination

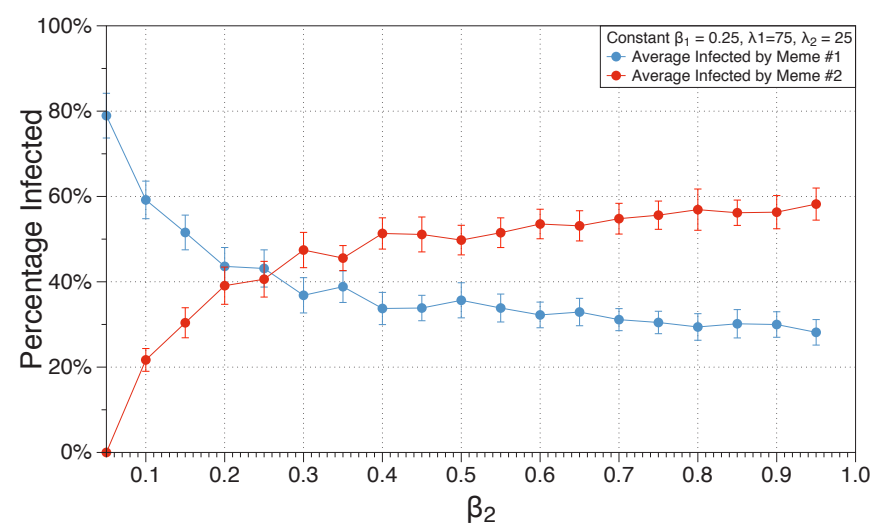

(b) Cross-Contamination vs. $\beta_{2}$

Fig. 6. Cross contamination simulation results.

\section{RELATED WORK}

We now proceed to reviewing related work in the context of single-meme and multiple-meme propagation from epidemiol- ogy, communication networks, game theory, and data mining literature.

Single-meme propagation. Many works focus on single meme propagation on one single topology. Compartmental 
models like SIS, SIR, etc., have been well-studied in many epidemiological texts [9], [1], [24]. The evolution of blogs and the maximization of influence propagation are studied in [3], [25]. Information cascades models are proposed to study the meme propagation in word-of-mouth communications [26], [2]. Richardson et al. proposed a meme propagation model to achieve optimal viral marketing plans [27]. Numerous studies exist on virus propagation on the Internet based on the basic epidemic models of infection [4], [5]. Virus propagation under special cases have been studied, e.g., in IPv6 Internet [28]. A fundamental question in epidemiology is the presence of a threshold, under which an epidemic is guaranteed not to happen. Pastor-Satorras et al. [10] proposed an epidemic threshold condition for random power law networks, which uses the "mean-field" approach. Ganesh et al. [13] and Yang et al. [11] provided epidemic threshold for the single-virus on single topology. Prakash et al. [12] gave the epidemic threshold condition for almost all single-virus epidemic models on a single static network. Cohen et al. [21] studied the well-known acquaintance immunization method and showed that it is much better than random methods. Tong et al. [22] provide a simple greedy $(1-1 / e)$ approximation algorithm for immunizing nodes under the SIS model.

Multiple memes and interdependent networks. Newman [14] studied multiple viruses on a single, special random graph and provided the epidemic threshold for the case when the second virus propagates over the residual network after the propagation of the first virus has completed. This scenario is close to the dynamics of propagation of a single virus - one virus passed over the network, the second virus starts to pass over the residual network. Models for multiple cascades have been studied as extensions of the independent cascade model, where once a node is infected with a cascade, it never change its state [29]. Multiple viruses propagation on simple fair-play single network was investigated [30]. The effects of cascades in inter-dependent networks (e.g., Internet router and power electricity networks) were investigated by Buldyrev et al. [31]. However, all of these works are completely different from our problem as we consider the more realistic and challenging scenario of competing memes propagating simultaneously on composite networks. In this paper, we have significantly extended our preliminary work [16] by: (a) proposing and evaluating an effective prediction scheme, Eigenpredictor, (b) considering immunization strategies with various suppression techniques to affect the outcome of the propagation, and (c) evaluating the effect of cross-contamination across various graphs.

Game theory. Meier et al. [32] studied inoculation games in social networks, where each node selfishly decides whether or not to protect itself. The game between a virus and an alert over a network was investigated by Aspnes et al. [33]. Kostka et al. [34] studied competing campaigns as a game-theoretical problem and showed that being the first player was not always advantageous. However, these works using game theory are different from our problem where we assume that all nodes are passive and follow the same propagation model.

\section{CONCLUSION}

In this paper, we have designed an effective methodology, EigenPredictor, to predict which meme will eventually prevail. Our theoretical and experimental results show that EigenPredictor achieves very high accuracy (above 95\%) on a wide variety of real and synthetic datasets. Given the outcomes predicted by our EigenPredictor, we have designed and evaluated various suppression schemes to alter the results of the competing memes on composite networks. Extensive experimental results have revealed the comparative effectiveness of suppression schemes. Finally, we formulate and provide an initial study of the effect of cross-contamination across the composite graphs, where a meme cross-over and starts propagating on the other network's topology.

\section{ACKNOWLEDGEMENTS}

We would like to thank the anonymous reviewers for their feedback. This material is based upon work supported by the National Science Foundation under Grants No.IIS1017415, CNS-1064646 and NSF CISE NECO-0832069, and by DARPA SMISC W911NF-12-C-0028 and ARL W911NF-092-0053. This work is also partially supported by funds from the VT College of Engineering.

\section{REFERENCES}

[1] A. G. McKendrick, "Applications of mathematics to medical problems," Edin. Math. Society, 1926.

[2] J. Goldenberg, B. Libai, and E. Muller, "Talk of the network: A complex systems look at the underlying process of word-of-mouth," Marketing Letters, 2001

[3] D. Gruhl, R. Guha, D. Liben-Nowell, and A. Tomkins, "Information diffusion through blogspace," in $W W W, 2004$.

[4] S. Staniford, V. Paxson, and N. Weaver, "How to 0wn the Internet in Your Spare Time," in USENIX Security Symposium, 2002.

[5] R. W. Thommes and M. J. Coates, "Epidemiological Modelling of Peerto-Peer Viruses and Pollution," in IEEE INFOCOM, 2006.

[6] E. M. Rogers, Diffusion of Innovations, 5th Edition. Free Press, August 2003.

[7] N. Ungerleider, "Massive Egyptian Protests Powerd by YouTube, Twitter, Facebook, Twitpic," in Fast Company, 2011.

[8] H. W. Hethcote, "The mathematics of infectious diseases," SIAM Review, vol. 42, 2000.

[9] R. M. Anderson and R. M. May, Infectious diseases of humans: Dynamics and control. Oxford Press, 2002.

[10] R. Pastor-Satorras and A. Vespignani, "Epidemic dynamics in finite size scale-free networks," Phys. Rev. E, vol. 65, 2002.

[11] Y. Wang, D. Chakrabarti, C. Wang, and C. Faloutsos, "Epidemic spreading in real networks: an eigenvalue viewpoint," in IEEE SRDS, 2003.

[12] B. A. Prakash, D. Chakrabarti, M. Faloutsos, N. Valler, and C. Faloutsos, "Threshold conditions for arbitrary cascade models on arbitrary networks," IEEE ICDM, 2011.

[13] A. Ganesh, L. Massoulie, and D. Towsley, "The Effect of Network Topology in Spread of Epidemics," in IEEE INFOCOM, 2005.

[14] M. E. J. Newman, "Threshold effects for two pathogens spreading on a network," Physical Review Letters, 2005.

[15] F. Li, Y. Yang, and J. Wu, "CPMC: An Efficient Proximity Malware Coping Scheme in Smartphone-based Mobile Networks," in IEEE INFOCOM, 2010.

[16] X. Wei, N. Valler, B. A. Prakash, I. Neamtiu, M. Faloutsos, and C. Faloutsos, "Competing Meme Propagation on Networks: A Case Study of Composite Networks," in ACM CCR, 2012.

[17] M. W. Hirsch and S. Smale, Differential Equations, Dynamical Systems and Linear Algebra. Academic Press, 1974.

[18] A.-L. Barabási and R. Albert, "Emergence of scaling in random networks," Science, 1999.

[19] A. Sala, L. Cao, C. Wilson, R. Zablit, H. Zheng, and B. Y. Zhao, "Measurement-calibrated graph models for social network experiments," in $W W W, 2010$ 
[20] D. W. Hosmer and S. Lemeshow, Applied Logistic Regression. John Wiley, 1989.

[21] R. Cohen, S. Havlin, and D. ben Avraham, "Efficient immunization strategies for computer networks and populations," Physical Review Letters, 2003.

[22] H. Tong, B. A. Prakash, C. E. Tsourakakis, T. Eliassi-Rad, C. Faloutsos, and D. H. Chau, "On the vulnerability of large graphs," in IEEE ICDM, 2010.

[23] J. O. Kephart and S. R. White, "Directed-Graph Epidemiological Models of Computer Viruses," IEEE S\&P, 1991.

[24] $\_$"Measuring and modeling computer virus prevalence," in IEEE $S \& P, 1993$

[25] D. Kempe, J. Kleinberg, and E. Tardos, "Maximizing the spread of influence through a social network," in ACM SIGKDD, 2003.

[26] S. Bikhchandani, D. Hirshleifer, and I. Welch, "A theory of fads, fashion, custom, and cultural change in informational cascades," J. Political Economy, 1992.

[27] M. Richardson and P. Domingos, "Mining knowledge-sharing sites for viral marketing," in ACM SIGKDD, 2002.

[28] Z. Chen and C. Ji, "Measuring Network-Aware Worm Spreading Ability," in IEEE INFOCOM, 2007.

[29] C. Budak, D. Agrawal, and A. E. Abbadi, "Limiting the Spread of Misinformation in Social Networks," in WWW, 2011.

[30] B. A. Prakash, A. Beutel and C. Faloutsos., "Winner-takes-all: Competing Viruses on fair-play networks," in $W W W, 2012$.

[31] S. V. Buldyrev, R. Parshani, G. Paul, H. E. Stanley, and S. Havlin, "Catastrophic cascade of failures in interdependent networks," Nature, 2010.

[32] D. Meier, Y. A. Oswald, S. Schmid, and R. Wattenhofer, "On the windfall of friendship: inoculation strategies on social networks," in ACM EC, 2008.

[33] J. Aspnes, N. Rustagi, and J. Saia, "Worm versus alert: Who wins in a battle for control of a large-scale network?" in OPODIS, 2007.

[34] J. Kostka, Y. Oswald, and R. Wattenhofer, "Word of mouth: Rumor dissemination in social networks," in SIROCCO, 2008.

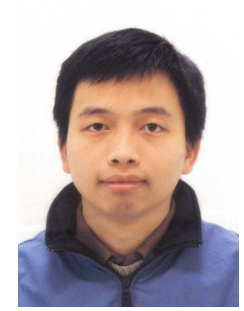

Xuetao Wei is a Ph.D candidate in the department of computer science and engineering at University of California, Riverside. His research interests span the areas of systems, security and networks with an emphasis on smartphones, network science, and green networks and systems.

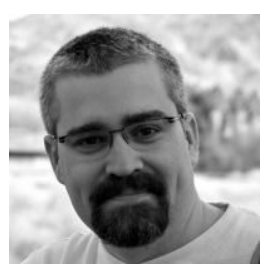

Nicholas Valler has received his Ph.D. from the University of California, Riverside in the Summer of 2012. His research interests include network science, network operations, and data mining applications. $\mathrm{He}$ currently works for CrowdCompass, Inc., in Portland, Oregon as a DevOps Engineer.

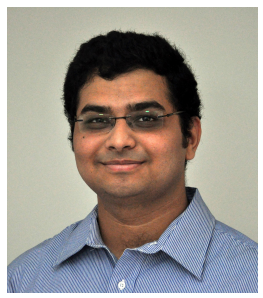

B. Aditya Prakash is an Assistant Professor in the Computer Science Department at Virginia Tech. He graduated with a Ph.D. from the Computer Science Department at Carnegie Mellon University in 2012, and got his B.Tech (in CS) from the Indian Institute of Technology (IIT) - Bombay in 2007. He has published 22 refereed papers in major venues and holds two U.S. patents and has given two tutorials (VLDB 2012 and ECML/PKDD 2012). His work has received one best paper award and two bestof-conference selections (CIKM 2012, ICDM 2012, ICDM 2011). His interests include Data Mining, Applied Machine Learning and Databases, with emphasis on large real-world networks and time-series.

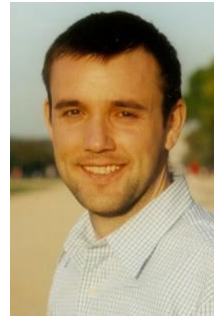

Iulian Neamtiu is an assistant professor in the Department of Computer Science and Engineering at the University of California, Riverside. He received his Ph.D. from the University of Maryland, College Park in 2008. His research interests are in programming languages, software engineering, and the smartphone side of systems and security. He is a recipient of the NSF CAREER award.

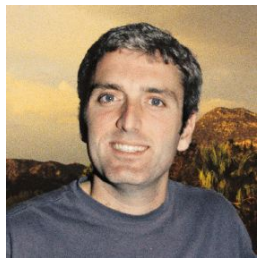

Michalis Faloutsos is a faculty member of the Computer Science Dpt in the University of New Mexico since 2012. He got his bachelor's degree at the National Technical University of Athens and his M.Sc and Ph.D. at the University of Toronto. His interests include, Internet protocols and measurements, peer-to-peer networks, network security, BGP routing, and ad-hoc networks. With his two brothers, he co-authored the paper on power-laws of the Internet topology, which received the ACM SIGCOMM Test of Time award. His work has been supported by many NSF and military grants, for a cumulative total of more than $\$ 6$ million. Several recent works have been widely cited in popular printed and electronic press such as slashdot, ACM Electronic News, USA Today, and Wired. Most recently he has focused on the classification of traffic and web-security, and co-founded a cyber-security company in 2008, offering services as www.stopthehacker.com, which received two SBIR grants from the National Science Foundation, and institutional funding in Dec 2011.

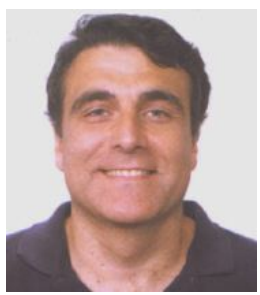

Christos Faloutsos is a Professor at Carnegie Mellon University. He has received the Presidential Young Investigator Award by the National Science Foundation (1989), the Research Contributions Award in ICDM 2006, the SIGKDD Innovations Award (2010), nineteen "best paper" awards (including two "test of time" awards), and four teaching awards. He is an ACM Fellow, he has served as a member of the executive committee of SIGKDD; he has published over 200 refereed articles, 11 book chapters and one monograph. He holds six patents and he has given over 30 tutorials and over 10 invited distinguished lectures. His research interests include data mining for graphs and streams, fractals, database performance, and indexing for multimedia and bio-informatics data. 